\title{
VitroJet: advanced control and ease of use in cryo-EM sample preparation
}

Giulia Weissenberger ${ }^{1}$, Frank Nijpels ${ }^{1}$, Rene Henderikx ${ }^{2}$ and Bart Beulen ${ }^{2}$

${ }^{1}$ CryoSol World, Maastricht, Limburg, Netherlands, ${ }^{2}$ CryoSol World, United States

Over the past decade, cryo-electron microscopy (cryo-EM) became a powerful mainstream method in structural biology. In spite of the advances, cryo-EM remains challenging and expensive due to limited efficiency and reproducibility of sample preparation. The inconsistency in grid preparation has pushed for many developments in the recent years. The VitroJet, first developed in 2017, introduced and combined several new concepts in order to provide control over grid preparation.

Automation and control through innovative techniques have been key to VitroJet development and in standardization of grid preparation. The machine works with pre-mounted autogrids and has three key compartments providing flexibility for parameter optimization: an integrated plasma cleaner, a deposition chamber, and a cryogenic chamber. The custom-built plasma cleaner has been designed to ensure wettability between grids with optional gas inlets for custom gas mixtures. The sample is introduced in the deposition chamber where it is maintained at its dewpoint. The dewpoint loop prevents concentration of the sample by evaporation, enabling accurate layer control. Pin printing comprises a solid pin bearing a sub-nanoliter amount of sample. The pin is brought to a pre-determined gap from the grid forming a capillary bridge. Through a scribing motion of the pin, an instantaneous thin film is deposited that does not require additional thinning, e.g. by blotting, nanowires or evaporation. The quality of the deposited layer is assessed by a camera situated in the deposition chamber for evaluation of layer thickness and overall grid quality. The cryogenic chamber houses two ethane jets that are activated simultaneously to vitrify the autogrid, targeting the center first. This results in higher cooling rates and ensures vitreous ice in pre-mounted autogrids.

By the start of 2021, a number of commercial VitroJets have arrived to labs around the world. These machines are equipped to provide ease of use. Automation is achieved through handling up to twelve grids per session without any need of user interference. A twelve pin drum intended for deposition is placed into the device at the start and a new pin is switched, avoiding potential cross contamination. Lastly, the frozen autogrids are automatically stored in gridboxes under liquid nitrogen maintained at a constant level through an autofill mechanism. By keeping the heart of the machine in a conditioned environment throughout the session, reproducibility and different applications can be explored. Furthermore, grid quality along with sample quality can be evaluated without being bound to the microscope, saving expensive screening time. In the discussion, we will present results associated with pre-screening of protein samples on grids, the various applications of the VitroJet and updates from our first external collaborators.

Reference

Ravelli, Raimond BG, et al. "Cryo-EM structures from sub-nl volumes using pin-printing and jet vitrification." Nature communications 11.1 (2020): 1-9. 